\title{
Investigating the Characteristics and
} Evolution of Apical Membrane Antigen 1 (AMA1) of Plasmodium sp. using Phylogenetic Approach in Searching for Drug Candidate

\section{Rizky Nurdiansyah¹, Kevin Nathanael Ramanto¹, and Priscilla Jessica ${ }^{2}$}

1Department of Bioinformatics, School of Life Sciences, Indonesia International Institute for Life Sciences, Jakarta, Indonesia

${ }^{2}$ Department of Biotechnology, School of Life Sciences, Indonesia International Institute for Life Sciences, Jakarta, Indonesia

\section{Abstract}

Malaria is an endemic disease caused by Plasmodium parasite with female Anopheles mosquitoes as a vector. With the increase of drug resistance Plasmodium emerging around the world, a new method should be devised against the spread of Plasmodium $s p$ lineage. Protein evolution of Plasmodium $s p$. can be an invaluable aid as an

Corresponding Author: Rizky Nurdiansyah rizky.nurdiansyah@i3ı.ac.id

Received: 1 February 2020 Accepted: 8 February 2020 Published: 16 February 2020

Publishing services provided by Knowledge E

(c) Rizky Nurdiansyah et al. This article is distributed under the terms of the Creative Commons Attribution License, which permits unrestricted use and redistribution provided that the original author and source are credited.

Selection and Peer-review under the responsibility of the IC-BIOLIS Conference Committee. input for rational drug design and immune-informatics methods based on the novel virulent gene that exists in these protozoa. Previously, we did data mining in the PlasmoDB database and found several proteins shared by Plasmodium, one of them was the Apical Membrane Antigen 1 (AMA1). This protein was further analyzed for its characteristics and evolutionary properties. AMA1 protein sequences from human-infecting Plasmodium (P. falciparum, vivax, knowlesi, ovale, and malariae) and non-infectious ( $P$. berghei, coatneyi, and cynomolgi) were retrieved from PlasmoDB. Maximum likelihood phylogenetic tree with molecular clock analysis was reconstructed from AMA1 sequences using MEGAX. Protein domain analysis was done using the INTERPRO server. Several domains that can induce protective immunity and vaccine target were found in AMA1 of those plasmodium, such as coiled-coil, disordered region, and signal peptide domain. Furthermore, molecular clock analysis showed a similar evolutionary rate between AMA1 protein in human-infecting and non-infectious Plasmodium. Interestingly, the phylogenetic tree showed a mixed cluster of humaninfecting with non-infectious, indicating a unique evolutionary relationship between Plasmodium lineage. Thus, this information could be beneficial in developing the drug and vaccine for Plasmodium-related infection.

Keywords: AMA1, drug target, protein domain, protein evolution, Plasmodium.

\section{Introduction}

Malaria is a vector-borne disease caused by Plasmodium parasites that are commonly found in developing countries [1]. Even though the efforts to eradicate malaria and its 
spread are numerous, starting from net, insect repellent, prophylaxis, early diagnostics, and combination therapies, the case of malarial infection is still very high. World Health Organization (WHO) estimated 219 million malaria cases with 435 thousand of mortality cases in 2017 [2]. Drug resistance poses as one of the most urgent issues in malaria eradication. Artemisinin combined therapy ( $A C T)$, the recommended first line of treatment for malaria, has hit a roadblock of partial resistance in Western Cambodia region [3]. The partial resistance itself is marked by the delayed blood-stage parasite [4]. This event causes delayed parasite clearance, allowing a small amount of Plasmodium parasite to survive over the course of treatment. This little amount of survived parasite would trigger a recrudescence, interpreted as the condition in which artemisinin-based therapy fails to cure the patient with resistant parasite phenotype.

Nowadays, WHO recommends treatment for uncomplicated malaria with the following ACTs: artemether-lumefantrine, artesunate-amodiaquine, artesunate-mefloquine, artesunate-sulfadoxine-pyrimethamine, and dihydroartemisinin-piperaquine [5]. Firstly, one ACT will be used at a time in a country. When a high failure rate $(>10 \%)$ is recorded, then another ACT will be deployed in place of the failing ACT. Despite the utilization of such ACT system deployment, malaria is still highly prevalent in South East Asia region. One of the reasons being is all ACT used artemisinin or its derivatives, despite the rise of partial artemisinin resistance in Greater Mekong Subregion: Cambodia, Laos, Myanmar, Thailand, and Vietnam [4]. The resistance is known to have spread from Cambodia, as the first country to report ACT treatment failure, to surrounding countries [3], [4].

These findings indicate that the artemisinin resistance is persistent and does not develop at random readily. The rise on resistance against ACT might eventually lead to the development of complete artemisinin resistance parasite and persistent infection from insufficient treatment. For this reason, there is a need to further study another drug candidate. Evolutionary relationship of the plasmodium genome may shed a new perspective in looking for new target candidate.

Interestingly, several proteins were found to be shared among plasmodium species after data mining in PlasmoDB database (https://plasmodb.org/), one of them is apical membrane antigen 1 protein (AMA-1). The mature form of this protein is found in the merozoite surface and it is well-established that AMA-1 is one of the essential proteins for the malarial parasite to infect the red blood cells (RBC) [6], [7]. Several studies had developed substantial research regarding the potency of AMA-1 to be a vaccine candidate. Drew et al (2012) reported the antigenic diversity of P. falciparum AMA-1 which could give some challenges in designing the vaccine [8]. The structural approach had been established to identify the binding site of AMA-1 and vaccine design [9], 
[10]. The polymorphic feature of AMA-1 leads to a challenge in the process of vaccine design [8], [11]. The evolutionary relationship of the plasmodium species has been reported using several molecular markers [12]--[14], but the AMA-1 evolutionary relationship among the plasmodium species is not yet elucidated.

The objective of this study is to provide valuable information regarding the evolutionary relationship of AMA-1 protein by implementing the phylogenetic analysis from various species of Plasmodium. Furthermore, the characteristic differences of these proteins in each plasmodium were observed in 2D and 3D protein structure. The results obtained provided valuable information that can support the development of a malaria vaccine using AMA-1 protein.

\section{Methods}

\subsection{Sequence retrieval and Domain analysis}

PlasmoDB database (https://plasmodb.org/) was mined for the data in AMA-1 protein sequences for 8 species of plasmodium. Five of those could infect humans ( $P$. falciparum, vivax, knowlesi, ovale, and malariae), while the rest, which could not infect human but could only infect other species (P. berghei, coatneyi, and cynomolgi), served as the comparison for the human infecting ones. A total of 24 protein sequences were retrieved from the database (Table 1). The retrieved sequences were analyzed for its domain and structure using the Interpro server (https://www.ebi.ac.uk/interpro/beta/ search/sequence/).

\subsection{Phylogenetic reconstruction}

All of the phylogenetic reconstruction was based on Hall's protocols and performed using MEGA X program [15], [16]. Multiple sequence alignment was performed using the MUSCLE algorithm built in the MEGA X [17]. After that, the best maximum likelihood model was estimated and then used to create the $M L$ tree. Jones, Taylor, and Thornton with gamma distribution ( $\mathrm{JTT}+\mathrm{G}$ ) model was used to reconstruct the phylogenetic tree. Tree robustness test was performed using 1000 replication bootstraps. Validation test was performed by comparing the $\mathrm{ML}$ tree with other trees with different algorithms: neigbour-joining and minimum evolution; which also reconstructed using MEGA X. 
TABLE 1: Retrieved AMA-1 protein sequences from PlasmoDB.

Sequence code
P. berghei ANKA
P.knowlesi_strain_H
P.knowlesi_strain_Malayan
-Strain_Pk1_A
P._vivax_P01
P.vivax_Sal-1
P.falciparum_7G8
P.falciparum_CD01
P.falciparum_Dd2
P.falciparum_GA01
P.falciparum_GB4
P.falciparum_GN01
P.falciparum_HB3

Accesion Number
PBANKA_0915000
PKNH_0931500
PKNOH_S120150200
PVP01_0934200
PVX_092275
Pf7G8_110037300
PfCD01_110038900
PfDd2_110036700
PfGA01_110037700
PfGB4_110040000
PfGN01_110038000
PfHB3_110036900

Sequence code
P.falciparum_IT
P.falciparum_KEO1
P.falciparum_KHO1
P.falciparum_KHO2
P.falciparum_MLO1
P.falciparum_SDO1
P.falciparum_SNO1
P.falciparum_TG01
P._malariae_UG01
P.ovale_curtisi_GHO1
P.coatneyi_Hackeri
P.cynomolgi_strain_M

Accesion Number

PfIT_110038000

PfKE01_110038000

PfKH01_110037800

PfKH02_110038700

PfML01_110038300

PfSD01_110036100

PfSN01_110036600

PfTG01_110037900

PmUG01_09042600

PocGH01_09039800

PCOAH_00026700

PcyM_0938200

\subsection{Timetree reconstruction}

Timetree was reconstructed based on Mello's protocol with the reconstructed ML tree as the base [18]. The default parameter from time tree tools of MEGA X was used. The time tree calibration for several Plasmodium species was retrieved from http://timetree.org/ [19].

\section{Results and Discussion}

\subsection{Phylogenetic tree reconstruction}

The phylogenetic tree was reconstructed from 24 AMA-1 sequences, which came from various plasmodium species (Fig. 1). P. berghei was considered as the outgroup in the tree due to its closeness to the root judging from the tree topology. Surprisingly, the tree produced a similar topology using other algorithms, (Fig. 1b, 1c). This indicates the evolutionary hypothesis is similar within those algorithms. Several studies also reported the same topology as our tree although the molecular marker is different [12], [13]. The tight grouping, in terms of branch length, within the same species suggested that AMA1 is conserved to a certain degree intraspecies. But an extensive study is needed to clarify the degree of AMA-1 protein sequence conservation.

The P. falciparum AMA-1 protein was clustered into one clade in all of the reconstructed trees, indicating the highly conserved sequence in P. falciparum AMA-1. 
Although some reports stated the polymorphism of AMA-1 in this species is due to some evolutionary causes, the AMA-1 protein is still relatively conserved intraspecies [8], [11], [20]. Interestingly, the P. falciparum clade had a closer evolutionary relationship with $P$. berghei, which is not infectious to human (Fig. 1). P. berghei has been widely known to infect rodents and usually used as a proxy to study the malarial infection in rodents animal model [21], [22]. Several studies reported the positive results of the $P$. berghei ANKA expressed the gene from $P$. falciparum, like the p48/45 and PF83/AMA-1, indicating the similar expression system in both species [23], [24].

Another interesting result was observed in the clade of the $P$. knowlesi with $P$. coatneyi and $P$. vivax with $P$. cynomolgi. Both $P$. coatneyi and $P$. cynomolgi are infecting monkeys but the tree showed the close evolutionary relationship with human infecting plasmodium. Moreover, both clades shared the same ancestor with P. malariae and $P$. ovale (Fig. 1). These results suggest that the AMA-1 protein may not exclusively responsible for host species infection properties. 
A

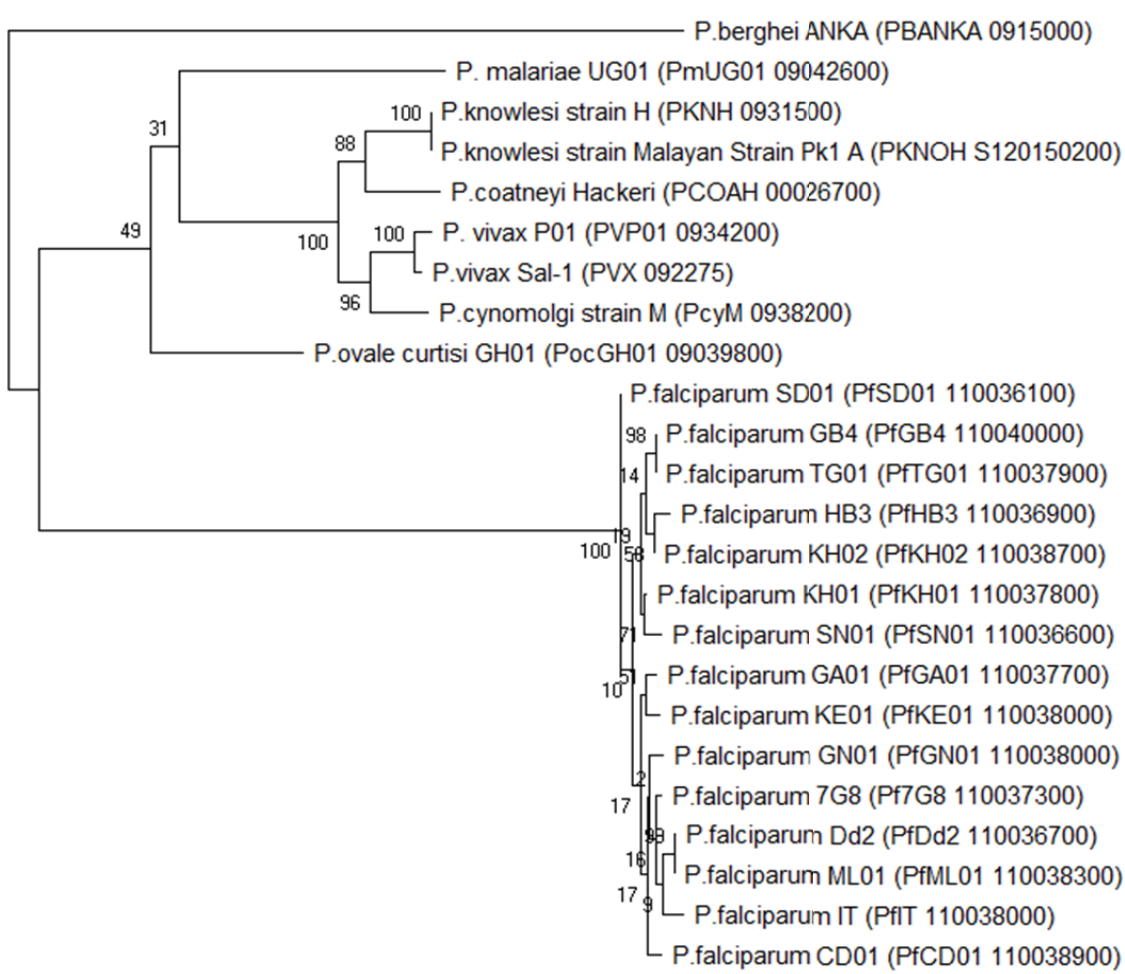

0.10

B

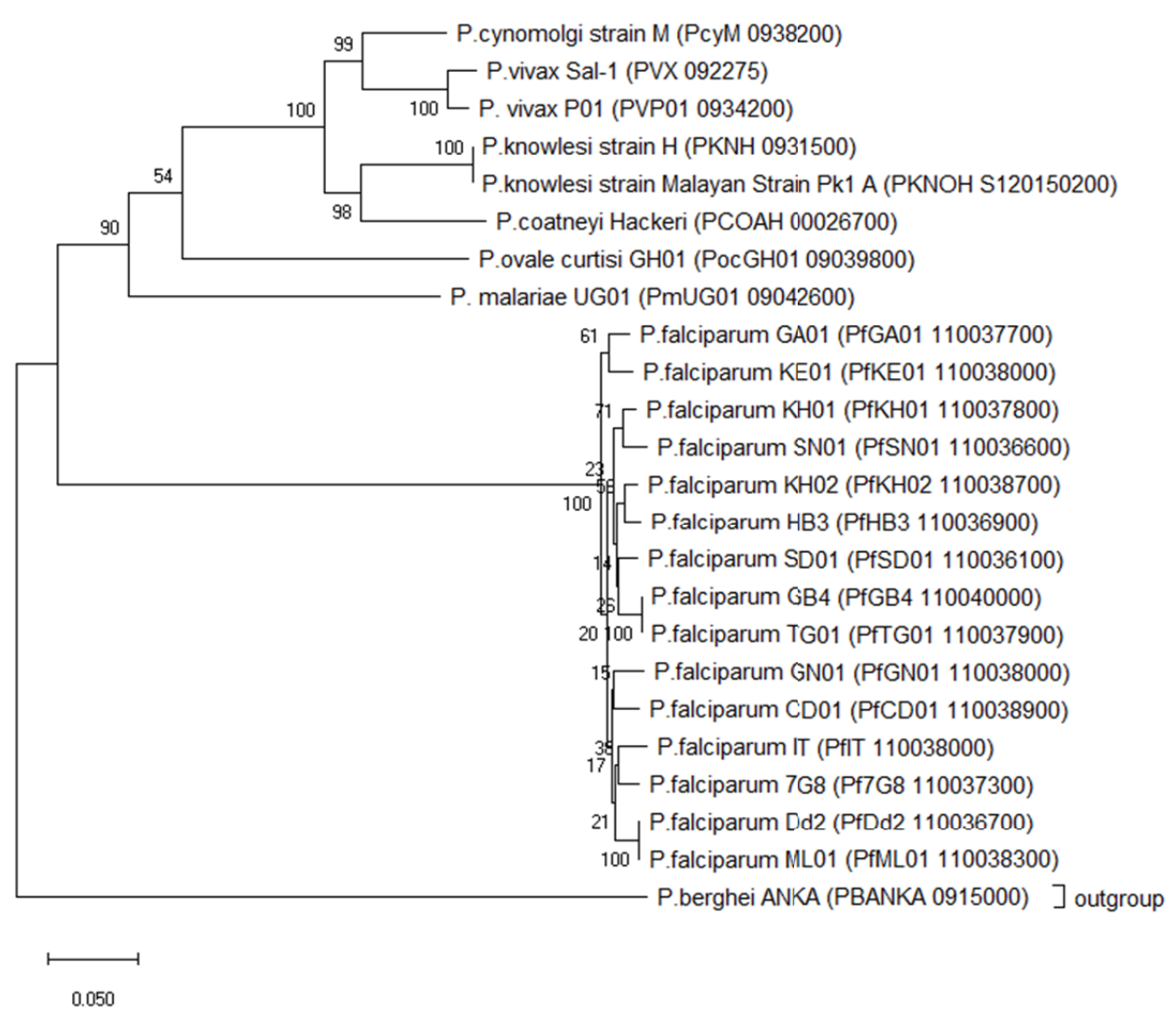




\section{C}

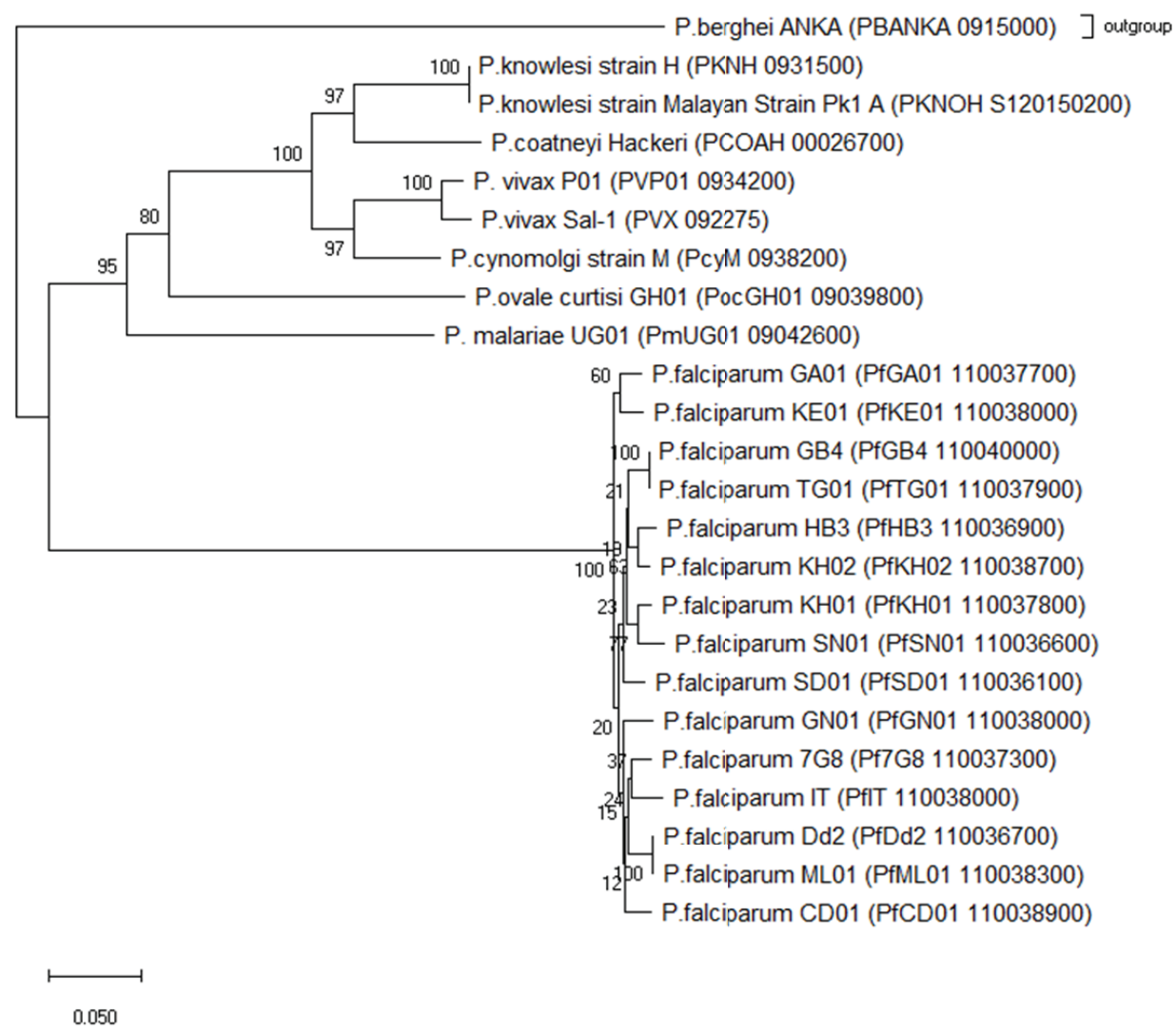

Figure 1: Reconstructed phylogenetic tree of AMA-1 protein from various plasmodium species. A. Maximum Likelihood tree with JTT $+\mathrm{G}$ model. B. Minimum evolution tree. C. Neighbor-joining tree. Every tree was reconstructed using 1000 bootstraps.

Both $P$. coatneyi and $P$. cynomolgi infect simians and show similar symptoms to the malarial infection to human. In details, $P$. coatneyi infection could induce severe anemia, coagulopathy, renal impairment, and metabolic dysfunction, similar to human cases of malaria [25]. An interesting study revealed that the genomes of $P$. knowlesi, $P$. vivax, $P$. coatneyi and $P$. cynomolgi were hypothesized coming from the similar ancestor and create the monkey clade in plasmodium phylogenetic tree [12], in accordance to our reported result in AMA-1 phylogenetic tree. The elucidation of the $P$. cynomolgi and $P$. coatneyi genomic sequences could serve a critical step in creating a model system to treat $P$. vivax, one of the major malaria agent outside Africa [12], [14]. It could also be true for AMA-1 protein vaccine design. 


\subsection{Time tree reconstruction}

The time tree was reconstructed from the $M L$ tree from the previous analysis. Based on the time tree, it was shown that some AMA-1 relative time divergence somehow supported the conserved hypothesis in the ML tree. Interspecies, the divergence is relatively narrow in the relative time divergence. The monkey clade of the $P$. knowlesi with $P$. coatneyi and P. vivax with P. cynomolgi was hypothesized to have the earliest divergence in 16.30 MYA. Our result is following the relative divergence of the genomics sequences [12]. Interestingly, the relative divergence time from $P$. falciparum with the monkey clades was shown to be a divergence in 77.85 MYA, indicating the AMA-1 protein sequence of $P$. falciparum is relatively different with the monkey clades and more closely related to the ancestor of AMA-1. Interestingly, $P$. falciparum and $P$. vivax AMA-1 structure have been reported to have similar structure even though their sequence is substantially different. Interestingly, the sequence could complement each other when combined, in terms of infecting host and avoiding the immune system, [26]. This report indicated the flexibility in retaining conserved structure in charge of infecting the host of AMA-1, which in turn could serve the ideal target for vaccine development.

A surprising result was observed in the placement of $P$. berghei AMA-1 in the time tree. Although previous trees showed that the $P$. berghei was on the outer clades and, hypothetically speaking, closer to the ancient AMA-1, the $P$. berghei was placed in the inner clades and closely related to $P$. ovale and diverged 25 MYA. This result is actually per another report using mitochondrial barcode sequence as the marker [13]. The report stated the $P$. ovale and $P$. berghei shared the same ancestry, while $P$. falciparum is the closest one to the outgroup.

\subsection{Domain and structure analysis}

To support the evolutionary relationship result, domain and structural analysis were done to elucidate the difference between the domain of AMA-1 of those plasmodium species. Due to the similarities of protein regions for each plasmodium strains within the same species, only the representative of each Plasmodium species were reported. Based on the result from InterPro, each of the protein belongs to the AMA-1 superfamily and has merozoitesa receptor proteins. Interesting result was found in the domain prediction of the AMA-1. Every AMA-1 protein had a similar pattern: starting from the signal peptide regions, followed by non-cytoplasmic domain, helix transmembrane region, and then the last part is the cytoplasmic domain of the protein. Surprisingly, 


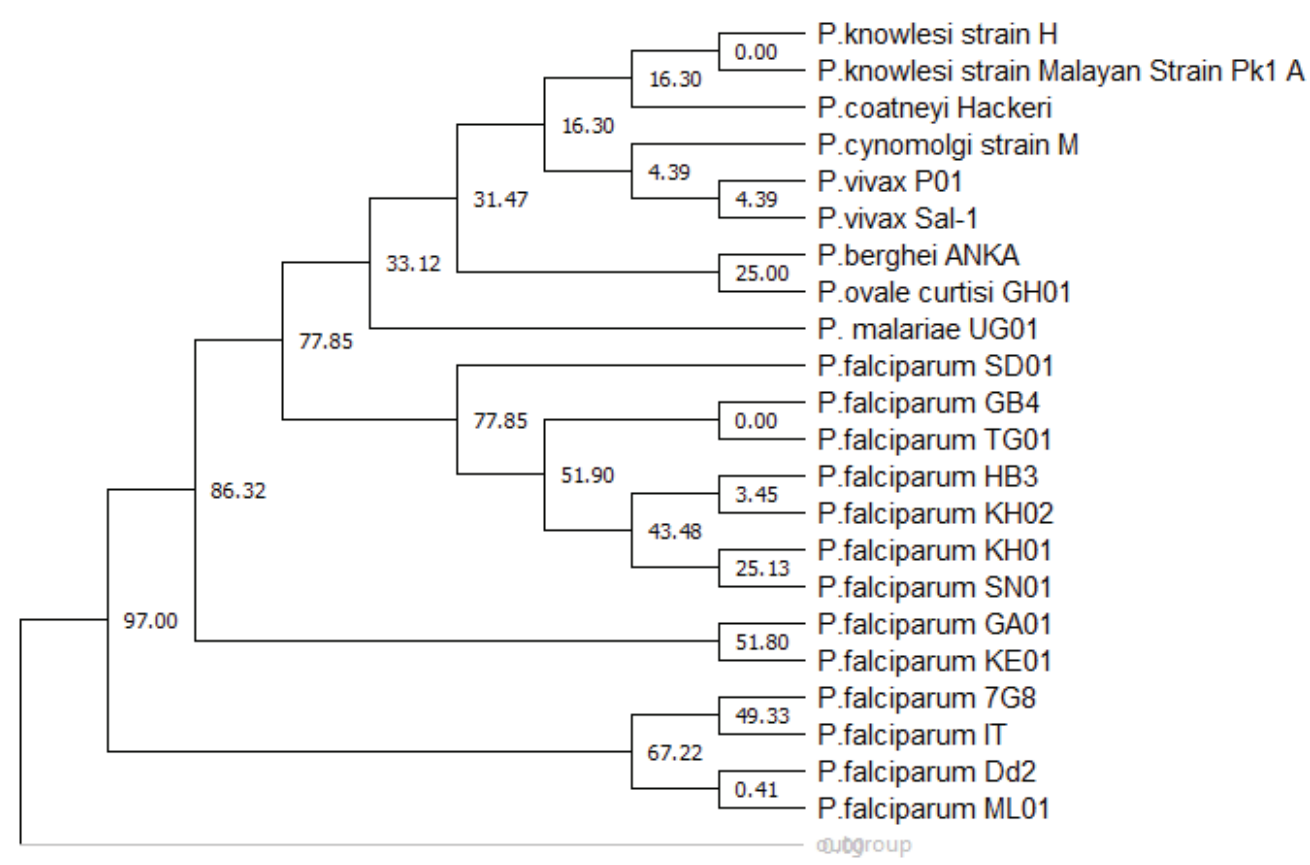

Figure 2: Time tree of AMA-1 protein divergence time. Numbers in each intersection indicates estimated divergence time in million years ago (MYA).

$P$. ovale and $P$. vivax were not predicted to have signal peptide regions, but it may be the prediction bias from the InterPro software (Fig. 3).

The previous report showed the flexibility in P. falciparum and P. vivax AMA-1 [26] yet the similar pattern in the regions of AMA-1 suggest some degree of structural conserveness in AMA-1 protein throughout the Plasmodium species. The similar pattern in the AMA-1 organization could serve as the ingredient for vaccine candidates. The signaling peptides have been studied as the candidate for a peptide-based vaccine to induce the immune system in targeting pathogen due to the similarity in structure and motifs [27].

Several unique patterns were observed as well, such as the coil region in the noncytoplasmic region of $P$. cynamolgi, P. knowlesi, $P$. ovale, and P. vivax. Disordered regions were found in the cytoplasmic regions of $P$. falciparum, $P$. vivax, and $P$. ovale, which all of them are well-known for its human infecting properties. These results are very interesting when compared to the phylogenetic tree. The coil region was observed in the clade of $P$. cynamolgi and $P$. vivax but not in its sister clade member, $P$. coatneyi. The coil regions are mostly observed in the human-infecting plasmodium, except $P$. falciparum. The disordered region was also mostly found in human-infecting plasmodium except for P. malariae (Fig. 3). 


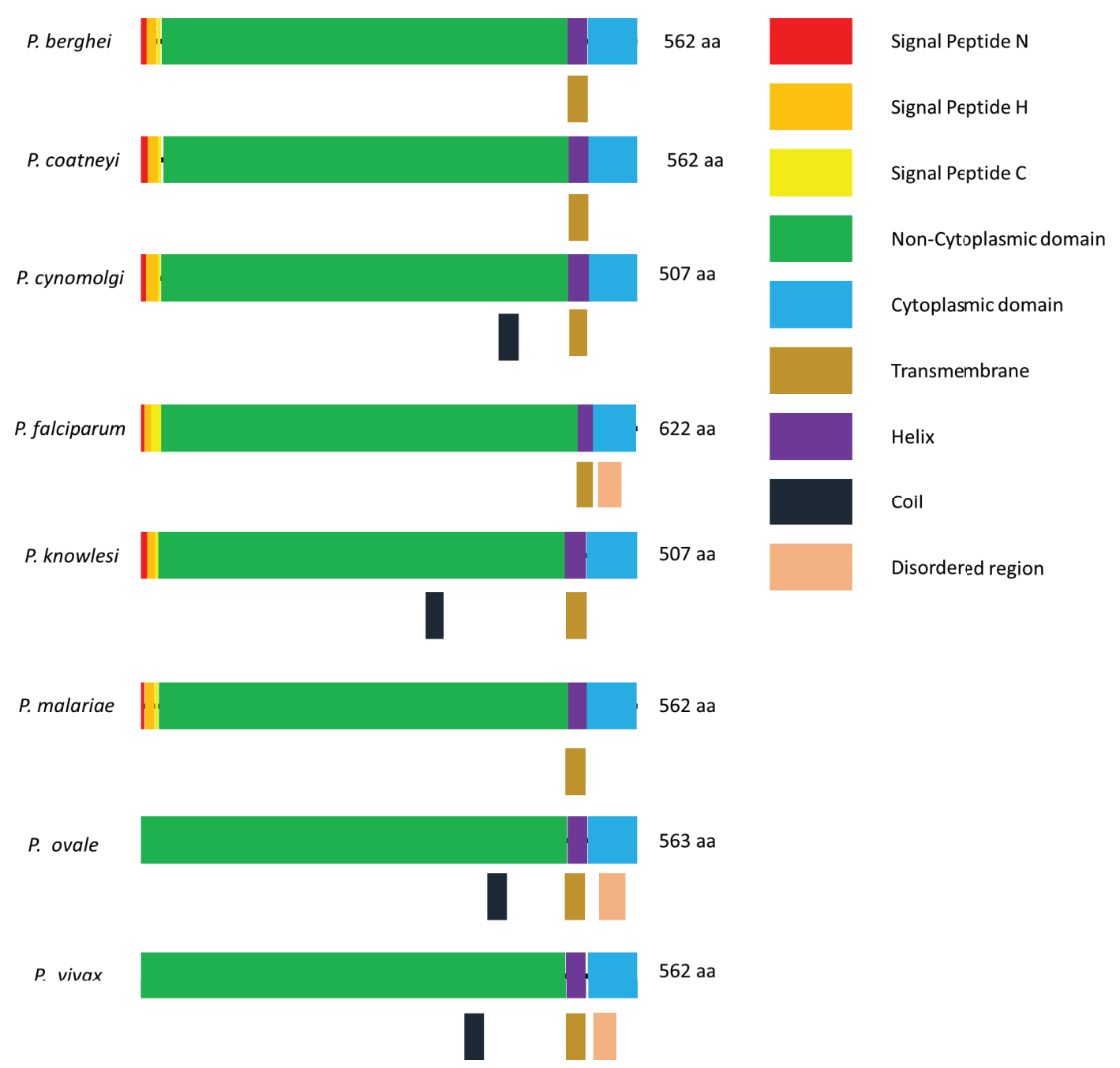

Figure 3: Interpro domain prediction analysis results from the representative sequences of each analyzed Plasmodium species.

These unique patterns could serve some pointers in the AMA-1 vaccine design. $P$. vivax and $P$. cynomolgi are clustered into one clade and both have the coil region in the non-cytoplasmic domain. The unique coil pattern in the protein organization may serve as the target for the same clade plasmodium. Several reports indicated the usage of coil regions in vaccine development in the P. falciparum [28], [29]. Surprisingly, the InterPro analysis didn't show any coil region in the $P$. falciparum but it had a disordered region in the cytoplasmic domain. The disordered region is the elusive part of the protein and it is well known for modulating the protein's function and enabling the protein to be involved in many cellular processes [30], [31]. The dynamic structure of this region is also contributing to its complexity [32] and even to the degree of oncogenicity [33]. The significance of the disordered region in protein is well understood which could serve as the target for drugs [30]. In addition to most of the human infecting plasmodium had a disordered region in their organization, this part could serve as another target. However, 
due to the position of the region is in the cytoplasmic domain, some modification in the vaccine would be necessary to ensure the vaccine delivery.

\section{Conclusion}

This study elucidates some interesting aspects of AMA-1 protein characteristics and evolution in several Plasmodium species. The evolutionary relationship of AMA-1 is hypothesized to not following its infectious route to human, even though the protein itself is responsible for its infection to the host cell. The protein domain prediction found the coil region in the non-cytoplasmic region and the signal peptides and disordered region in the cytoplasmic domain could be a beneficial target for the vaccine in human-infecting plasmodium. However, substantial continuation studies are needed to elucidate the vaccine candidate potential for AMA-1. Nevertheless, this study could serve as the initial step for the fine-grained AMA-1 vaccine design for plasmodium.

\section{Acknowledgments}

The authors send the sincerest thanks to $\mathrm{I} 3 \mathrm{~L}$ research and community service department (LPPM) for their full support to this research and Giovanni Wijaya from Global Sevilla School for his contribution for the study during his summer internship in i3L.

\section{Conflict of Interest}

The authors declare no conflict of interest in this research.

\section{Supporting Grants}

The research is funded by DRPM DIKTI funding with the scheme "Penelitian Dosen Pemula" in 2019 funding year (Contract No: 48/AKM/MONOPNT/2019).

\section{References}

[1] A. Y. Vittor et al., "Linking Deforestation to Malaria in the Amazon: Characterization of the Breeding Habitat of the Principal Malaria Vector, Anopheles darlingi," Am J Trop Med Hyg, vol. 10, no. 1, pp. 54--56, 2013.

[2] World Health Organization, World Malaria Report 2018. 2018. 
[3] H. Noedl, Y. Se, K. Schaecher, B. L. Smith, D. Socheat, and M. M. Fukuda, "Evidence of artemisinin-resistant malaria in Western Cambodia," N. Engl. J. Med., vol. 359, no. 24, pp. 2619--2620, 2008.

[4] R. Amato et al., "Origins of the current outbreak of multidrug-resistant malaria in southeast Asia: a retrospective genetic study," Lancet Infect. Dis., vol. 18, no. 3, pp. 337--345, 2018.

[5] World Health Organization, "Artemisinin resistance and artemisinin-based combination therapy efficacy," 2018.

[6] G. H. Mitchell, A. W. Thomas, G. Margos, A. R. Dluzewski, and L. H. Bannister, "Apical Membrane Antigen 1, a Major Malaria Vaccine Candidate, Mediates the Close Attachment of Invasive Merozoites to Host Red Blood Cells," Infect. Immun., vol. 72, no. 1, pp. 154--158, 2004.

[7] T. Triglia et al., "Apical membrane antigen 1 plays a central role in erythrocyte invasion by Plasmodium species," Mol. Microbiol., vol. 38, no. 4, pp. 706--718, 2000.

[8] D. R. Drew et al., "Defining the Antigenic Diversity of Plasmodium falciparum Apical Membrane Antigen 1 and the Requirements for a Multi-Allele Vaccine against Malaria," PLoS One, vol. 7, no. 12, 2012.

[9] F. Jahangiri, N. Jalallou, and M. Ebrahimi, "Analysis of Apical Membrane Antigen (AMA)-1 characteristics using bioinformatics tools in order to vaccine design against Plasmodium vivax," Infect. Genet. Evol., vol. 71, no. March, pp. 224--231, 2019.

[10] M. Akter et al., "Identification of the Binding Site of Apical Membrane Antigen 1 (AMA1) Inhibitors Using a Paramagnetic Probe," ChemMedChem, vol. 1, 2019.

[11] A. Ebrahimzadeh, A. Gharaei, and K. Saryazdi, "Allelic Diversity of Polymorphic AMA-1 (Apical Membrane Antigen 1) Vaccine Candidate Antigen of Plasmodium falciparum in Two Population of Imported and Indigenous Cases in South-East of Iran using Nested-PCR and RFLP," J. Trop. Dis., vol. 02, no. 05, pp. 5--8, 2014.

[12] A. Gilabert et al., "Plasmodium vivax-like genome sequences shed new insights into Plasmodium vivax biology and evolution," PLoS Biol., vol. 16, no. 8, pp. 1--25, 2018.

[13] G. G. Rutledge et al., "Plasmodium malariae and P. ovale genomes provide insights into malaria parasite evolution," Nature, vol. 542, no. 7639, pp. 101--104, 2017.

[14] S. Tachibana et al., "Plasmodium cynomolgi genome sequences provide insight into Plasmodium vivax and the monkey malaria clade," vol. 44, no. 9, pp. 1051--1055, 2013.

[15] B. G. Hall, "Building phylogenetic trees from molecular data with MEGA," Mol. Biol. Evol., vol. 30, no. 5, pp. 1229--1235, 2013. 
[16] S. Kumar, G. Stecher, M. Li, C. Knyaz, and K. Tamura, "MEGA X: Molecular evolutionary genetics analysis across computing platforms," Mol. Biol. Evol., vol. 35, no. 6, pp. 1547--1549, 2018.

[17] R. C. Edgar, "MUSCLE: Multiple sequence alignment with high accuracy and high throughput," Nucleic Acids Res., vol. 32, no. 5, pp. 1792--1797, 2004.

[18] B. Mello, "Estimating timetrees with MEGA and the timetree resource," Mol. Biol. Evol., vol. 35, no. 9, pp. 2334--2342, 2018.

[19] S. B. Hedges, J. Marin, M. Suleski, M. Paymer, and S. Kumar, "Tree of life reveals clock-like speciation and diversification," Mol. Biol. Evol., vol. 32, no. 4, pp. 835--845, 2015.

[20] A. G. Craig et al., "The role of animal models for research on severe malaria," PLoS Pathog., vol. 8, no. 2, 2012.

[21] R. Basir et al., "Plasmodium berghei ANKA infection in ICR mice as a model of cerebral malaria," Iran. J. Parasitol., vol. 7, no. 4, pp. 62--74, 2012.

[22] A. L. Goodman et al., "The utility of Plasmodium berghei as a rodent model for anti-merozoite malaria vaccine assessment," Sci. Rep., vol. 3, 2013.

[23] C. H. M. Kocken et al., "Precise timing of expression of a Plasmodium falciparumderived transgene in Plasmodium berghei is a critical determinant of subsequent subcellular localization," J. Biol. Chem., vol. 273, no. 24, pp. 15119--15124, 1998.

[24] A. S. Othman et al., "Expression of full-length Plasmodium falciparum P48/45 in P. berghei blood stages: A method to express and evaluate vaccine antigens," Mol. Biochem. Parasitol., vol. 224, no. July, pp. 44--49, 2018.

[25] A. Moreno et al., "Plasmodium coatneyi in rhesus macaques replicates the multisystemic dysfunction of severe malaria in humans," Infect. Immun., vol. 81, no. 6, pp. 1889--1904, 2013.

[26] D. R. Drew, P. R. Sanders, G. Weiss, P. R. Gilson, B. S. Crabb, and J. G. Beeson, "Functional Conservation of the AMA1 Host-Cell Invasion Ligand between P. falciparum and P. vivax: A Novel Platform to Accelerate Vaccine and Drug Development," J. Infect. Dis., vol. 217, no. 3, pp. 498--503, 2018.

[27] R. Kovjazin and L. Carmon, "The use of signal peptide domains as vaccine candidates," Hum. Vaccines Immunother., vol. 10, no. 9, pp. 2733--2740, 2014.

[28] V. Villard et al., "Rapid identification of malaria vaccine candidates based on $\alpha$-helical coiled coil protein motif," PLoS One, vol. 2, no. 7, 2007.

[29] C. P. Karch et al., "The use of a P. falciparum specific coiled-coil domain to construct a self-assembling protein nanoparticle vaccine to prevent malaria," J. Nanobiotechnology, vol. 15, no. 1, pp. 1--10, 2017. 
[30] M. M. Babu, "The contribution of intrinsically disordered regions to protein function, cellular complexity, and human disease," Biochem. Soc. Trans., vol. 44, no. 5, pp. 1185--1200, 2016.

[31] M. Madan Babu, R. Van Der Lee, N. Sanchez De Groot, J. Rg Gsponer, J. Gough, and K. Dunker, "Intrinsically disordered proteins: regulation and disease This review comes from a themed issue on Sequences and topology Edited," Curr. Opin. Struct. Biol., vol. 21, pp. 1--9, 2011.

[32] J. H. Na, W. K. Lee, and Y. G. Yu, "How do we study the dynamic structure of unstructured proteins: A case study on nopp140 as an example of a large, intrinsically disordered protein," Int. J. Mol. Sci., vol. 19, no. 2, 2018.

[33] E. R. Tamarozzi and S. Giuliatti, "Understanding the role of intrinsic disorder of viral proteins in the oncogenicity of different types of HPV," Int. J. Mol. Sci., vol. 19, no. 1, 2018. 